Letter to the Editors,

Dear Editors,

\section{FLEXOR HALLUCIS LONGUS MUSCLE ATROPHY DUE TO A CHRONIC COMPARTMENT SYNDROME OF THE LOWER LEG}

We would like to report a case of chronic compartment syndrome in a long distance runner.

The leg has four compartments - anterior, lateral, superficial and deep posterior - which are prone to produce ischaemic damage to muscle and nerves when intractable swelling is present. The clinical picture of the acute compartment syndrome comprises symptoms such of pain, pallor, paresthesia, pulselessness and finally, paralysis. The syndrome in its chronic form is seen in long distance runners and other endurance athletes and it differs from the acute compartment syndrome in having only some of the classical features. The pathology in chronic compartment syndrome is due to non-yielding compartmental boundary formed by an excessively tight fascia causing abnormally high tissue pressure at rest. The pressure is further increased by muscle activity to a point that interferes with muscle blood flow during intensive activity and the metabolic demands of the tissues are no longer met (Martens et al, 1984).

The potential sequelae of a compartment syndrome include persistent anaesthesia and motor weakness (Sheridan and Matsen, 1976), contractures (Clark et al, 1978), renal failure (Mubarak and Owen, 1975) and even death (Lonka and Pedersen, 1987). The best method of prevention of these sequelae is early diagnosis and treatment.

A 26-year-old competitive long distance runner exhibited signs of medial tibial compartment syndrome during the winter season when training on a slippery road surface a year before admission to our clinic. The first subjective sign had been mild pain along the medial edge of tibia. A few days later severe pain developed posterior to the medial malleolus and disappeared two weeks later while the patient had a week's rest. Later some discomfort was present along the medial edge of the tibial shaft but not in the malleolar area during training. The patient experienced change in the co-ordination when running; the foot seemed clumsy on landing and slightly weak on take-off. A month after the initial symptoms a hollow could be observed at the medial side of tibia.

The patient had first been treated with anti-inflammatory drugs and physiotherapy without relief of symptoms. On admission no tightness of the calf muscles was observed. There was no tenderness and $X$-ray did not reveal a stress fracture. A hollow was present on the medial side of the tibia in the deep posterior compartment and nuclear magnetic resonance imaging at $0.02 \mathrm{~T}$ (Acutscan, Instrumentarium Corp.) using $T_{2}$-weighted and $T_{1}$-weighted pulse sequences indicated atrophy of the flexor hallucis longus muscle without change in the relaxation times or spin density. Thus replacement of muscle by scar or fatty tissue was excluded (Fig.). Electromyography showed polyphasia of the flexor hallucis longus muscle while all the other leg muscles were electromyographically normal. Since there was no indication for fasciotomy (Sheridan and Matsen, 1976) the patient was treated conservatively. Muscle rehabilitation was initiated with isometric work for seven to ten days and isotonic exercises were instituted thereafter. After general cardiorespiratory exercises the

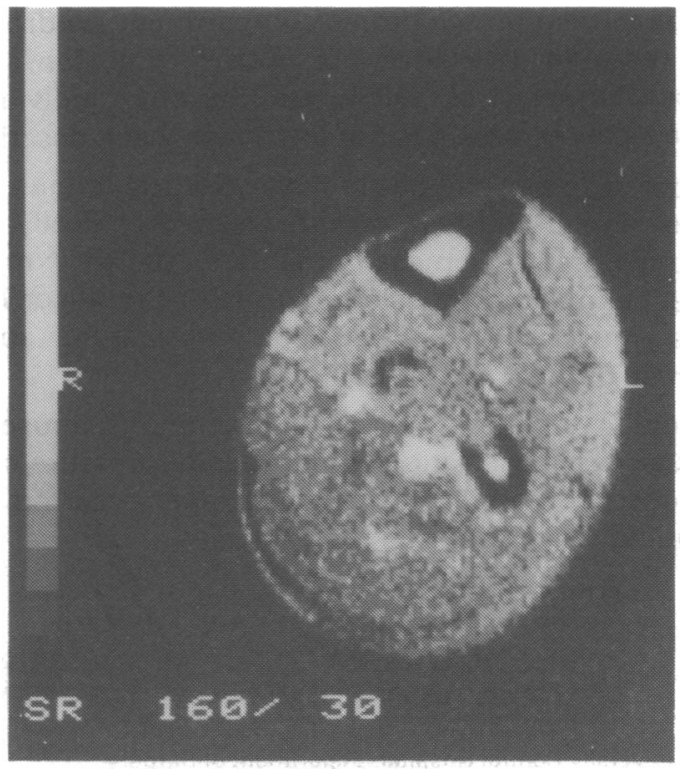

Nuclear magnetic resonance imaging at the level of the hollow at the media side of the tibia indicated atrophic changes of the flexor hallucis longus muscle. In the schematic diagram the location of the atrophic muscle is pointed out (asterisk). The four compartments - deep posterior (DP) superficial posterior (SP), lateral (L) and anterior (A) as well as tibia (T) and fibula (F) - are shown.

patient was capable of resuming full training and no recurrence of the medial tibial syndrome occurred despite continuing running. The hollow at the medial side of tibia became gradually more indistinct, and one and a half years after the onset of symptoms only a flat hollow was present as an indication of regeneration - obviously not complete - of the atrophied muscle. At that time EMG had turned to normal.

There are high demands for the control of the foot in running, which requires balanced synergistic interplay of muscles. Biomechanical faults may disturb the delicate balance of muscles and lead to overuse and fatigue of the muscular system. Continued physical stress at this point may lead to structural stress to bone with resultant periostitis and pain. Periosteal pain may require rest of the muscles and disuse atrophy may result. When increased compartmental pressure is present in addition there is risk of atrophic changes in the muscles being reinforced (Puranen and Alavaikko, 1981). Although several physical 
findings in relation to chronic compartment syndrome has been reported (weakness of muscle function, muscle hernias (Detmer et al, 1985)), atrophy of an individual leg muscle as in our case has not previously been described.

In our case atrophy of the flexor hallucis longus muscle had developed and was diagnosed both clinically and by electromyographic measurements. Nuclear magnetic resonance imaging revealed atrophy of the flexor hallucis longus muscle, while the leg musculature appeared otherwise normal.

Atrophy of the flexor hallucis longus muscle had obviously decreased the compartmental pressure since no recurrence of the medial tibial syndrome occurred despite continuing endurance running exercises.

Yours faithfully,

\section{LEHTO, MD* $\dagger$ \\ M. KORMANO, MD‡ \\ V. RANTAKOKKO, MD* \\ M. JÄRVINEN, MD§}

*Turku University Central Hospital, Department of Surgery

tSports Medical Research Unit, University of Turku

¥Turku University Central Hospital, Department of Radiology

$\S$ Tampere University Central Hospital, Department of Surgery
Present address for correspondence:

Matti Lehto, MD

Tampere University Central Hospital

Department of Surgery

SF-33520 Tampere

FINLAND

\section{References}

Clark, M. W., D’Ambrosia, R. D. and Roberts, J. M., 1978 “Equinus contracture following Bryant's traction". Orthopaedics 1: 311-312.

Detmer, D. E., Sharpe, K., Sufit, R. L. and Girdley, F. M., 1985 "Chronic compartment syndrome: diagnosis, management and outcomes". Am.J. Sports Med. 13: 162-170.

Lonka, L. and Pedersen, R. S., 1987 "Fatal rhabdomyolysis in marathon runner". Lancet 1: 857-858.

Martens, M. A., Backaert, M., Vermaut, G. and Mulier, J. C, 1984 "Chronic leg pain in athletes due to a recurrent compartment syndrome". Am.J.Sports Med. 12: 148-151.

Mubarak, S. J. and Owen, C. A., 1975 "Compartmental syndrome and its relation to the crush syndrome: a spectrum of disease. A review of 11 cases of prolonged limb compression". Clin.Orthop.Relat.Res. 113: 81-89.

Puranen, J. and Alavaikko, A., 1981 "Intracompartmental pressure increase on exertion in patients with chronic compartment syndrome in the leg". J.Bone Jt.Surg. 63A: 1304-1309.

Sheridan, G. W. and Matsen, F. A., 1976 "Fasciotomy in the treatment of the acute compartment syndrome". J.Bone Jt.Surg. 58A: 112-115.

\section{Letter to the Editors,}

\section{Dear Editors,}

\section{EXAMINATION IN SPORTS MEDICINE}

The Society of Apothecaries of London are contemplating instituting an examination in Sports Medicine and the Master of the Society has asked me to produce proposals for consideration by the Society. I am interested in receiving a wide spectrum of opinion about such an examination. I would be grateful if any reader who has ideas or wishes to be consulted about such an examination could write to me at Westminster Hospital, London SW1P 2AP.

Yours faithfully,

F. B. GIBBERD, MD, FRP

Westminster Hospital

London SW1P 2AP 